\title{
Temperature control in very low birthweight infants during first five days of life
}

\author{
A J Lyon, M E Pikaar, P Badger, N McIntosh
}

\begin{abstract}
Aim-To determine ranges for skin temperatures in infants weighing under $1000 \mathrm{~g}$ in the first five days of life.

Method-Abdominal skin and foot temperatures were automatically collected each second, averaged over 1 minute and stored on computer. A computer program analysed the data in 83 babies weighing under $1000 \mathrm{~g}$ at birth over the first five days of life and expressed the temperatures as means and standard deviation. The temperature patterns seen in these babies were also visually analysed. The relation between an increasing abdominal skin-foot temperature difference and other signs of hypovolaemia was also studied.

Results-These babies all had similar temperature patterns. Just after birth there was little ability to vasoconstrict in the presence of cold stress and the babies behaved more like poikilothermic animals. Vasomotor tone developed in the first three days, resulting in a stabilisation of the abdominal skin temperature to a mean of $36.9^{\circ} \mathrm{C}$ and a widening of the central-peripheral temperature difference (Td) to a mean of $1.0^{\circ} \mathrm{C}$. A Td of > $2^{\circ} \mathrm{C}$ was associated with other evidence of hypovolaemia for only $11 \%$ of the time. Conclusions-Infants weighing under $1000 \mathrm{~g}$ have poor vasomotor control at birth and are at increased risk from cold stress. After the first two to three days of life, monitoring the central-peripheral temperature difference gives an early indication of cold stress. (Arch Dis Child 1997;76:F47-F50)
\end{abstract}

Keywords: temperature control; central-peripheral temperature difference; hypovolaemia.

Neonatal Unit, Simpson Memorial Maternity Pavilion, Edinburgh AJ Lyon

ME Pikaar

P Badger

Department Child Life and Health, University of Edinburgh

N McIntosh

Correspondence to: Dr AJ Lyon

Neonatal Unit

Simpson Memorial

Maternity Pavilion

Lauriston Place

Edinburgh
EH3 9YW.

Accepted 5 November 1996 and evaporation. The effect of these four modes will depend on gestation, postnatal age, the characteristics of the environment and the proportions of the body in contact with the mattress, and clothing. To determine the heat exchange between the infant and the environment we must know the heat loss from the skin per unit surface area, the total body surface area, and the proportion of the surface area participating in the different modes of heat exchange.

Individual studies have shown us the importance of all these factors in heat exchange and have led to recommendations about the physical environment of the preterm baby - for example, the importance of high humidity to prevent evaporative heat losses. ${ }^{45}$ However, the relation of the infant, and in particular the preterm baby, with its environment is constantly changing and although we understand something of this, what we need is an easy method of continuously monitoring and assessing the thermal state of the baby.

There is no simple cotside method of measuring heat exchange. Similarly, the metabolic effects, measured by oxygen consumption, cannot be monitored continuously in babies undergoing intensive care. Measurement of the baby's temperature is normal practice in neonatal care. However, the intermittent measurement of a single temperature tells us only how efficient the baby is at maintaining temperature but nothing about the metabolic cost of achieving it.

An early response to thermal stress is a change in peripheral temperature that occurs before any alteration in the central temperature. Cold stress causes peripheral vasoconstriction and centralisation of the circulation in an attempt to maintain the core temperature. Measurement of both a central and a peripheral temperature and the continuous monitoring of the central-peripheral temperature difference will give an early indication of thermal stress, before any change is seen in the core temperature. ${ }^{6}$ There is, however, little information on the normal temperatures of extremely preterm infants.

All babies in our intensive care unit have continuous monitoring of a central temperature with a probe on the abdominal skin, sited over the liver, and peripheral temperature measured on the sole of the foot. The aim of this study was to determine the effect of postnatal age on the abdominal and foot temperatures and the central-peripheral temperature difference in infants weighing less than $1000 \mathrm{~g}$ at birth. 


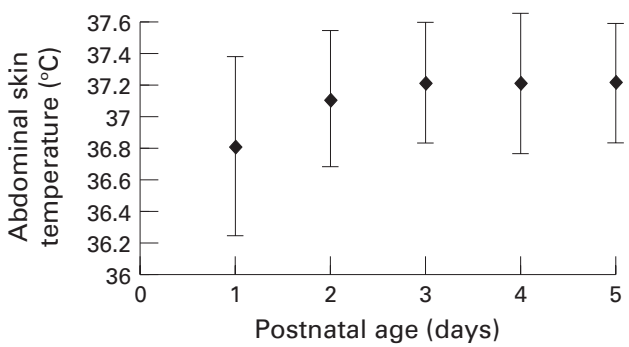

Figure 1 Mean (SD) abdominal skin temperature according to postnatal age

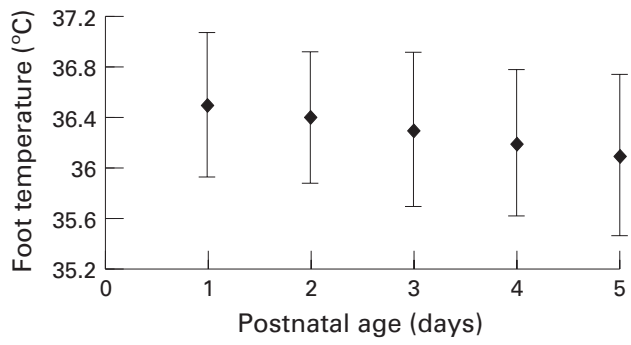

Figure 2 Mean (SD) foot temperature according to postnatal age

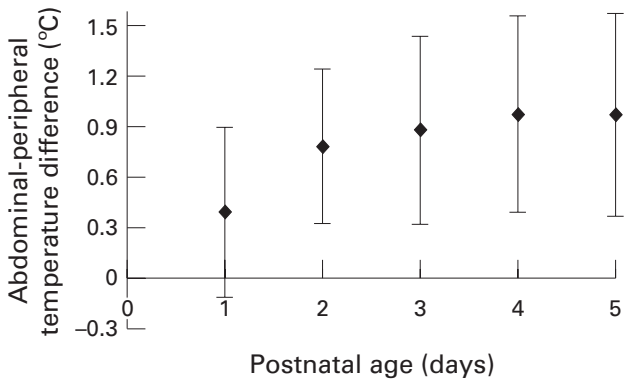

Figure 3 Mean (SD) central-peripheral temperature difference according to postnatal age

\section{Methods}

Babies weighing less than $1000 \mathrm{~g}$ admitted over a two year period, and with temperature data in the first five days of life, were included. A standard neonatal monitoring system (Hewlett-Packard, HP78344A) with two temperature channels was used. The temperature probes from this monitor were taped to the abdominal skin over the liver and to the sole of the foot and were covered to prevent any effect from direct heat. A computer monitoring system ${ }^{7}$ collected all physiological data, including both temperatures, at one second intervals from the monitor, averaged the readings over one minute, and stored this value. The data were also displayed as trend graphs on the screen at the cotside.

The babies were covered by a plastic sheet and nursed in humidified incubators $(80 \%$ relative humidity) for the first week of life. The incubators were used with air mode control, the nurses setting the temperature to achieve a neutral thermal environment. ${ }^{8}$

The temperature readings for each baby were analysed using a computer program which also removed artefact caused by the probes not in contact with the skin. For each baby the readings were averaged over each of the five days. These daily averages were then combined for all the babies giving an average temperature for each of the first five days of life. Comparison between the days was done by two-way analysis of variance. Stepwise linear regression was used to analyse any association between changes in the central-peripheral temperature difference $(\mathrm{Td})$ and outcome.

The computer program developed for the analysis contained a language which could be used to describe patterns within the data and could therefore be used to find developing trends. The relation between $\mathrm{Td}$ and hypovolaemia was tested by searching for episodes in which $\mathrm{Td}$ was increasing above $2^{0} \mathrm{C}$ in association with a rising heart rate and/or falling blood pressure. The proportion of time that a central-peripheral temperature difference of more than $2^{\circ} \mathrm{C}$-without any fall in the central temperature - was associated with these classic indicators of hypovolaemia, was calculated.

\section{Results}

Eighty three babies were included. Figures 1-3 and table 1 show the means and standard deviations of the abdominal (Tc), foot (Tp), and central-peripheral temperature difference (Td) for each postnatal day.

Table 2 gives the percentage of time that the abdominal skin temperature ( $\mathrm{Tc}$ ) was above $37.5^{\circ} \mathrm{C}$ or below $36.3^{\circ} \mathrm{C}$. The widest variations in this temperature were seen on day 1. Table 2 also gives the percentage of time that the central-peripheral temperature difference (Td) was above $2^{\circ} \mathrm{C}$ (a wide gap) or below $0^{\circ} \mathrm{C}$ - that is, the foot temperature was greater than that of the abdomen. This was not due to probe problems or other artefact, but was a real phenomenon, lasting anything from a few minutes to several hours. There seemed to be an association between death and the proportion of time that $\mathrm{Tp}$ was greater than $\mathrm{Tc}(\mathrm{P}=0.04)$, but this was no longer significant when birthweight was included in the regression analysis.

All the babies had a similar pattern in their temperature traces during the first two to three days of life. A typical example of the computer monitoring trace is shown in fig 4. Just after birth there was a period (A) when Tc and Tp were almost equal and were very variable, possibly responding to changes in the environmental temperature. There was little evidence of

Table 1 Mean (SD) abdominal skin (Tc), foot $(T p)$ and central-peripheral temperature difference with postnatal age

\begin{tabular}{lllllll}
\hline Postnatal age (days) & Tc mean $\left({ }^{\circ} C\right)$ & $T c S D$ & $T p$ mean $\left({ }^{\circ} C\right)$ & $T p S D$ & Td mean $\left({ }^{\circ} C\right)$ & $T d S D$ \\
\hline 1 & 36.8 & .56 & 36.5 & .57 & 0.4 & .51 \\
2 & 37.1 & .43 & 36.4 & .52 & 0.8 & .46 \\
3 & 37.2 & .38 & 36.3 & .61 & 0.9 & .56 \\
4 & 37.2 & .44 & 36.2 & .58 & 1.0 & .58 \\
5 & 37.2 & .38 & 36.1 & .64 & 1.0 & .60
\end{tabular}

Tc: (day 1 vs day 2)and (day 1 vs day 5) $-\mathrm{P}<0.0001$. Td: (day 1 vs day 2) and (day 2 vs day 5) $-\mathrm{P}<0.0001 . \mathrm{Tp}$ : (day 1 vs day 5 ) $-\mathrm{P}=0.001$. 
Table 2 Percentage of time abdominal skin temperature $(T c)>37.5$ and $<36.3^{\circ} \mathrm{C}$ and central-peripheral temperature difference $>2.0$ and $<0^{\circ} \mathrm{C}$

\begin{tabular}{lllll}
\hline Postnatal age (days) & $T c \%>37.5^{\circ} \mathrm{C}$ & $T c \%<36.3{ }^{\circ} \mathrm{C}$ & $\mathrm{Td} \%>2.0{ }^{\circ} \mathrm{C}$ & $\mathrm{Td} \%<0{ }^{\circ} \mathrm{C}$ \\
\hline 1 & 16.7 & 12.2 & 2.7 & 18.3 \\
2 & 13.9 & 6.3 & 4.3 & 9.8 \\
3 & 14.8 & 8.1 & 4.8 & 7.3 \\
4 & 8.0 & 7.1 & 6.4 & 5.5 \\
5 & 14.4 & 8.6 & 7.2 & 4.9 \\
\hline
\end{tabular}

peripheral vasoconstriction in response to cold stress and the foot temperature (Tp) was often greater than that of the abdominal skin (Tc). In phase $B$ the more normal pattern with $\mathrm{Tc}>\mathrm{Tp}$ was seen, but there were still frequent episodes of temperature reversal, often associated with handling. Phase $\mathrm{C}$ was associated with the development of normal peripheral vasoconstriction in response to cold stress and occurred at a median time of 29 hours after birth, with a range of 3 to 92 hours.

A central-peripheral temperature difference (Td) of more than $2^{\circ} \mathrm{C}$ was associated with a rising heart rate and/or falling blood pressure for only $11.4 \%$ of the total time. At all other times there was no other evidence to suggest hypovolaemia despite a high $\mathrm{Td}$.

\section{Discussion}

The continuous monitoring of a central and a peripheral temperature and the graphical display of the central-peripheral difference gives an early indication of developing cold stress in a neonate. The foot is traditionally used to measure peripheral temperature and in term babies this may be the only area of skin to react to increasing cold with vasoconstriction. ${ }^{9}$ Blood flow to the trunk may actually increase as the environmental temperature is lowered, ${ }^{9}$ emphasising the vulnerability of the exposed newborn baby to cold stress-a situation likely to be exacerbated by prematurity. As the

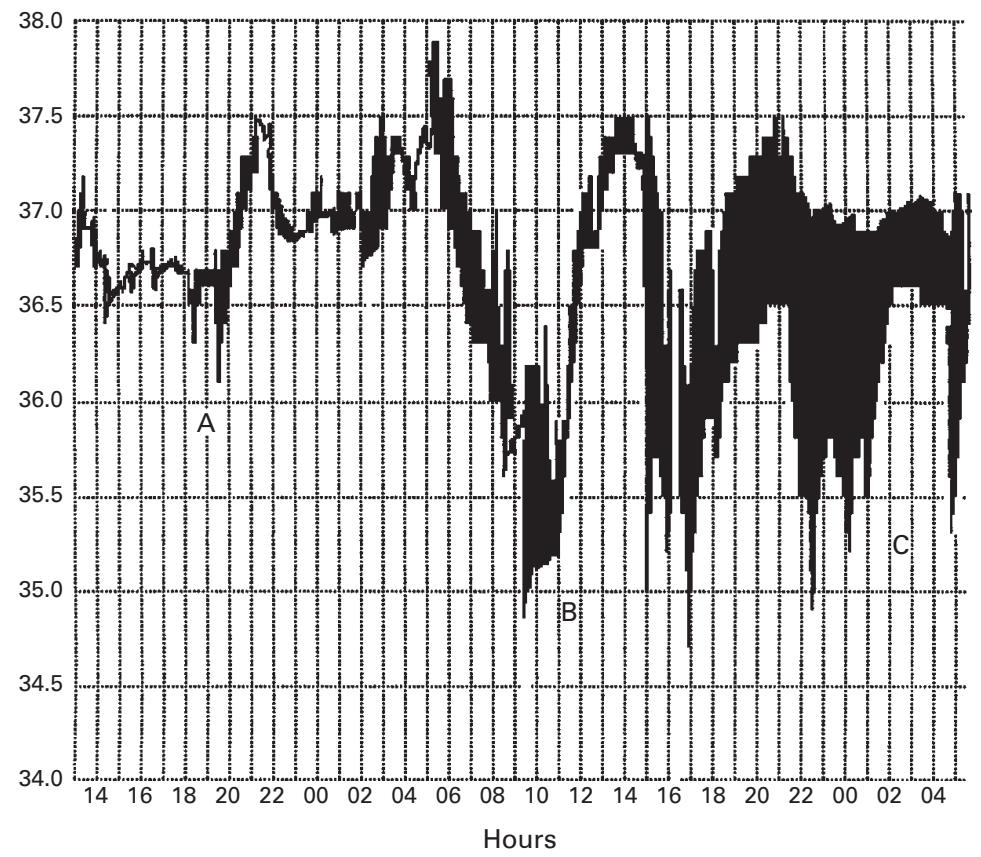

Figure 4 Computer monitor trace of abdominal skin (top line) and foot temperatures (bottom line), with shaded area showing central-peripheral temperature difference: baby boy, gestation 26 weeks, birthweight $700 \mathrm{~g}$, first 36 hours of life. abdominal skin does not vasoconstrict it can be used as an indicator of central temperature and is easier and safer than using the rectum, ${ }^{10-11}$ but the probes need to be screened from direct heat sources, such as phototherapy units. The babies were all nursed in humidified incubators and air mode control was used because of the known increased thermal stress caused by Servo control driven by a set abdominal skin temperature. ${ }^{12}$

The results are based on a large number of data points, and represent the ranges of temperatures achieved by these babies when cared for by experienced staff who altered incubator air temperature to maintain them in a state of optimal comfort. It is difficult to know how these relate to "normal temperatures" for this group of babies, but such normal ranges probably cannot be defined in a conventional way because of methodological problems in measuring heat flux and metabolic rates. Current published data are for more mature infants. ${ }^{8}$ Even if we had information on optimal environmental settings it would be important to have some means of continuously monitoring these babies as their relation with the environment changes. ${ }^{13}$ The use of the centralperipheral temperature difference gives a better indication of the thermal state of the baby than measurement of a single temperature alone.

Very preterm babies lose a significant amount of heat from the skin, particularly through evaporation, and their thermoregulatory mechanisms are immature. ${ }^{2}$ Peripheral vasoconstriction is an early reaction to cold stress but our results show that in infants weighing under $1000 \mathrm{~g}$ at birth, this response is not present at birth but develops over the first two to three days of life. Before this the central and peripheral temperatures are similar and both are directly affected by the environmental temperature - that is, the baby behaves more like a poikilotherm. As the baby develops the ability to vasoconstrict, the central temperature (Tc) is stabilised and the mean centralperipheral temperature difference $(\mathrm{Td})$ increases, suggesting better thermal stability. The apparent temperature instability in the first days of life was not associated with any other events in the baby, such as intraventricular haemorrhage.

During the first days of life, there were periods in which the foot was warmer than the abdominal skin $(\mathrm{Tp}>\mathrm{Tc})$ and this temperature reversal was often associated with handling. These babies were nursed in humidified incubators and with handling the humidity dropped rapidly as the incubator ports were opened. During this time evaporative heat losses would increase from exposed surfaces, such as the trunk. Often the baby's feet would remain covered during this handling and the slower heat loss from the feet compared with the trunk led to the temperature reversal. The association between death and the increased proportion of time with the reversed temperature pattern may just be a reflection of the fact that the sicker babies are handled more often.

In addition to being seen in cold stress, a high central-peripheral temperature difference 
might be the result of poor peripheral perfusion caused by hypovolaemia. A low circulating blood volume will result in a compensatory rise in heart rate and eventually to a fall in blood pressure. In this study these indirect indicators of hypovolaemia were only found in $11.4 \%$ of the total time that $\mathrm{Td}$ was greater than $2^{\circ} \mathrm{C}$, suggesting that changes in the centralperipheral temperature difference are more likely to reflect thermal stress in these babies.

Immature babies have a reduced vasomotor response to cold stress in the first days of life. The ability to vasoconstrict peripherally develops during the first postnatal days but before this time the infant is at increased risk from heat loss. Subsequently, the use of a centralperipheral temperature difference $(\mathrm{Td})$ is a useful indicator of cold stress and the aim should be to keep $\mathrm{Td}$ at around $1^{0} \mathrm{C}$ by altering incubator air temperature and humidity.

1 Sinclair JC. Management of the thermal environment. In:Sinclair JC, Bracken MB, eds. Effective care of the newborn infant. Oxford:Oxford University Press, 1992:4058.
2 Brück K. Neonatal thermal regulation. In: Polin R, Fox WW, eds. Fetal and neonatal physiology. Philadelphia, WB WW, eds. Fetal and neonatal

3 Houdas Y, Ring EF. Human body temperature. Its measurement and regulation. London: Plenum Press, 1982. 4 Silverman WA, Blanc WA. The effect of humidity on survival of newly born premature infants. Pediatrics 1957;20:477-87.

5 Sauer PJJ, Dane HJ, Visser HKA. Influence of variations in ambient humidity on insensible water loss and thermoneutral environment of low birth weight infants. Acta Paediatr Scand 1984;73:615-9.

6 Simbruner G. Temperature measurements and distribution of temperatures throughout the body in neonates. In: Okken A, Koch J, eds. Thermoregulation of sick and low birth Okken A, Koch J, eds. Thermoregulation of sick
weight neonates. Berlin: Springer 1995:53-62.

7 McIntosh N, Ducker DA, Bass CA. MARY - a computerised neonatal cot monitoring system. Intensive Therapy and Clinical Monitoring 1989;67:122-5.

8 Hey EN, Katz G. The optimum thermal environment for naked babies. Arch Dis Child 1970;45:328-34.

9 Karlsson H, Hänel S-E, Nilsson K, Olegård R. Measurement of skin temperature and heat flow from skin in term newborn babies. Acta Paediatr 1995;84:605-12.

10 Merenstein GB. Rectal perforation by thermometer. Lancet 1970;i:1007.

11 Morley CJ, Hewson PH, Thornton AJ, Cole TJ. Axillary and rectal temperature measurements in infants. Arch Dis and rectal temperatu

12 Ducker DA, Lyon AJ, Ross-Russell RR, Bass CA, McIntosh $\mathrm{N}$. Incubator temperature control effects on the very low birth weight infant. Arch Dis Child 1985;60:902-7.

13 Mok Q, Bass CA, Ducker DA, McIntosh N. Temperature instability during nursing procedures in preterm neonates. Arch Dis Child 1991;66:783-6. 